\title{
Substitution of TAG oil with diacylglycerol oil in food items improves the predicted 10 years cardiovascular risk score in healthy, overweight subjects
}

\author{
Vibeke H. Telle-Hansen ${ }^{1,2}$, Ingunn Narverud ${ }^{1,2}$, Kjetil Retterstøl ${ }^{2,3}$, Nima Wesseltoft-Rao ${ }^{1,2}$, \\ Annhild Mosdøl ${ }^{1}$, Linda Granlund ${ }^{4}$, Kirsti Forstrøm Christiansen ${ }^{4}$, Amandine Lamglait ${ }^{4}$, \\ Bente Halvorsen ${ }^{5}$, Kirsten B. Holven ${ }^{2}$ and Stine M. Ulven ${ }^{1}$ \\ ${ }^{1}$ Department of Health, Nutrition and Management, Faculty of Health Sciences, Oslo and Akershus University College of Applied Sciences, \\ Post Box 4, St. Olavsplass, 0130 Oslo, Norway \\ ${ }^{2}$ Department of Nutrition, Institute for Basic Medical Sciences, University of Oslo, Post Box 1046, Blindern, 0317 Oslo, Norway \\ ${ }^{3}$ Lipid Clinic, Medical Department, Rikshospitalet, Oslo University Hospital, Post Box 4950, Nydalen, 0424 Oslo, Norway \\ ${ }^{4}$ Mills DA, Post Box 4644, Sofienberg, 0506 Oslo, Norway \\ ${ }^{5}$ Research Institute for Internal Medicine, Faculty of Medicine, University of Oslo, Rikshospitalet, Oslo University Hospital, Post Box 4950, Nydalen, \\ 0424 Oslo, Norway
}

(Received 23 January 2012 - Final revision received 24 August 2012 - Accepted 4 September 2012)

Journal of Nutritional Science (2012), vol. 1, e17, page 1 of 13

doi:10.1017/jns.2012.18

\section{Abstract}

Dietary fat is normally in TAG form, but diacylglycerol (DAG) is a natural component of edible oils. Studies have shown that consumption of DAG results in metabolic characteristics that are distinct from those of TAG, which may be beneficial in preventing and managing obesity. The objective of the present study was to investigate if food items in which part of the TAG oil is replaced with DAG oil combined with high $\alpha$-linolenic acid (ALA) content would influence metabolic markers. A 12-week double-blinded randomised controlled parallel-design study was conducted. The participants $(n 23)$ were healthy, overweight men and women, aged $37-67$ years, BMI $27-35 \mathrm{~kg} / \mathrm{m}^{2}$, with waist circumference $>94 \mathrm{~cm}$ (men) and $>88 \mathrm{~cm}$ (women). The two groups received $20 \mathrm{~g}$ margarine, $11 \mathrm{~g}$ mayonnaise and $12 \mathrm{~g}$ oil per d, containing either high ALA and sn-1,3-DAG or high ALA and TAG. Substitution of TAG oil with DAG oil in food items for 12 weeks led to an improvement of the predicted 10 years cardiovascular risk score in overweight subjects by non-significantly improving markers of health such as total body fat percentage, trunk fat mass, alanine aminotransferase, systolic blood pressure, $\gamma$-glutamyl transferase, alkaline phosphatase and total fat-free mass. This may suggest that replacing TAG oil with DAG oil in healthy, overweight individuals may have beneficial metabolic effects.

Key words: Diacylglycerol: TAG: Overweight human subjects: Liver markers

Prevention and treatment of obesity and obesity-related diseases are major public health challenges ${ }^{(1-3)}$ and both the total amount of fat and the distribution of fat in the body are important determinants of the complications associated with obesity ${ }^{(4)}$. Surplus energy intake is stored as TAG in adipose tissue either as subcutaneous or visceral fat. Visceral fat depots, located in the trunk surrounding the organs, are recognised to be a more active endocrine organ, and contribute to an increased risk of metabolic complications, than subcutaneous fat depots $^{(4-7)}$. Hence, the endocrine activity of

Abbreviations: ALA, $\alpha$-linolenic acid; ALAT, alanine aminotransferase; ALP, alkaline phosphatase; ASAT, aspartame aminotransferase; CRP, C-reactive protein; DAG, diacylglycerol; FFM, fat-free mass; $\gamma$-GT, $\gamma$-glutamyl transferase; HOMA-IR, homeostatic model assessment for insulin resistance; hsCRP, high-sensitivity CRP.

* Corresponding author: Kirsten B. Holven, fax +47 22851341, email Kirsten.holven@medisin.uio.no 
visceral adipose tissue is an important factor in the development of the metabolic syndrome ${ }^{(8)}$, and central obesity is more strongly associated with increased risk of insulin resistance, the metabolic syndrome and CVD than BMI alone ${ }^{(9)}$. The endocrine activity of adipose tissue includes secretion of a number of adipokines (cytokines and hormones) leading to a pro-inflammatory state, and it has been shown that the reduction of visceral fat effectively prevents cardiovascular events ${ }^{(10)}$. Also, hepatic accumulation of fat is common in obese individuals and is associated with hepatic dysfunction ${ }^{(11)}$. Increased adipose tissue, and in particular visceral adipose tissue, is linked to increased levels of circulating liver function markers, such as alanine aminotransferase (ALAT) and $\gamma$-glutamyl transferase $(\gamma-G T)^{(12-16)}$.

Dietary fat is normally in TAG form, but diacylglycerol (DAG) is a natural component of various edible oils ${ }^{(17)}$ and is currently used in small quantities in foods as an emulsifier. Recent studies have shown that high consumption of DAG results in metabolic characteristics that are distinct from those of TAG, and that these characteristics may be beneficial in preventing and managing obesity. Experimental studies in human subjects have shown that DAG (mainly $s n-1,3-D A G$ ) decreases postprandial TAG compared with TAG controls ${ }^{(18,19)}$. Kamphuis et al. showed that meals rich in DAG increase fat oxidation $^{(20)}$. Dietary DAG has also been shown to reduce body fat (total, subcutaneous, visceral and hepatic fat mass) compared with $\mathrm{TAG}^{(21)}$, and consumption of DAG oil as part of a reduced-energy diet enhances loss of body weight and fat compared with TAG control oil ${ }^{(22)}$.

Vegetable oils such as flaxseed oil and rapeseed oils are the main dietary sources of the vegetable $n-3$ fatty acid $\alpha$-linolenic acid (ALA; $18: 3$ ). The relation between intake of ALA and CVD has been studied less extensively than the marine $n-3$ fatty acids and the relation is less clear. Some studies, including clinical trials, epidemiological investigations and experimental studies, have shown that intake of ALA has been beneficially associated with CVD risk ${ }^{(23)}$ or markers of risk ${ }^{(24)}$. Other studies have found no effect of ALA on CVD risk markers ${ }^{(25-28)}$.

The aim of the present study was to investigate whether intake of food items in which part of the TAG-based oil has been replaced with DAG combined with high ALA content would influence metabolic markers such as C-reactive protein (CRP), blood lipids, body composition, liver function markers and overall cardiovascular risk score in healthy, overweight individuals.

\section{Experimental methods}

\section{Subjects}

Healthy, overweight men and women who were willing to consume margarine, mayonnaise and oil daily for 14 weeks were recruited via advertisements in local newspapers from October 2008 to February 2009. The inclusion criteria were: age $18-70$ years, BMI $27-40 \mathrm{~kg} / \mathrm{m}^{2}$ and waist circumference $>94 \mathrm{~cm}$ for men and $>88 \mathrm{~cm}$ for women. Exclusion criteria were: type 2 diabetes mellitus, kidney-, liver-, gall bladder-, coronary-, endocrine- or chronic rheumatic disease, malign cancer during the last 5 years, hypertension $(\geq 160 / 100$ $\mathrm{mmHg}$ ), pregnancy and lactation. Regular use of antiinflammatory, lipid lowering and anti-hypertensive medications was not permitted. The present study was conducted according to the guidelines laid down in the Declaration of Helsinki and all procedures involving human subjects were approved by the Regional Committee of Medical Ethics (approval no. 6.2008.1368) and by the Norwegian Social Science Data Services (approval no. 19667). Written informed consent was obtained from all subjects. The study was registered at http://www.clinicaltrials.gov (no. NCT01034436).

\section{Study design}

The present study is part of a larger study involving healthy overweight subjects randomised to three different intervention groups: high ALA-DAG ( $n$ 10), high ALA-TAG (n 13) and low ALA-TAG ( $n$ 10) where the aim of the study was (1) to study the effect of replacing TAG-based food items with DAG-based food items and (2) to study the effect of low $v$. high ALA in TAG-based food items. In the present study, only data relating to the first aim are presented. In total, ninetysix subjects were screened for eligibility (minimum 4 weeks prior to the run-in period). For the present aim, forty-nine subjects were randomised and thirty-nine subjects received allocated interventions. Of the subjects, ten discontinued the intervention (declined to eat products, hospitalisation, lack of time and no explanation) and six had to be excluded from the analyses, leaving twenty-three subjects ( $n 10$ and 13) included in the final statistical analyses. A 12-week doubleblinded randomised controlled parallel-design study was conducted at Akershus University College from February to June 2009. Subjects were allocated to high ALA-DAG or high ALA-TAG further described in detail later. During a 2-week run-in period prior to the intervention period, all subjects included the same set of test products. The test products in the run-in period consisted of sunflower oil-based margarine $(20 \mathrm{~g})$ and mayonnaise $(11 \mathrm{~g})$ and sunflower oil $(12 \mathrm{~g})$. Fatty acids were given as TAG and the total amount of ALA in the run-in period was $0.15 \mathrm{~g}$ per $\mathrm{d}$.

At baseline, subjects were randomly assigned and stratified by sex into the intervention groups receiving products containing either high ALA $(8.29 \mathrm{~g} / \mathrm{d})$ and $s n-1,3-\mathrm{DAG}$ or high ALA $(8.51 \mathrm{~g} / \mathrm{d}$ ) and TAG (from now on referred to as the highALA-DAG group and high-ALA-TAG group, respectively) (Table 1). The test products were $20 \mathrm{~g}$ margarine, $11 \mathrm{~g}$ mayonnaise and $12 \mathrm{~g}(13 \mathrm{ml})$ oil per $\mathrm{d}$. Margarine was the only test product where $s n-1,3-\mathrm{DAG}$ oil was added. Rapeseed oil was used in mayonnaise as TAG $(8.84 \mathrm{~g} / \mathrm{d})$, and in the margarine either as $s n-1,3-D A G(11.2 \mathrm{~g} / \mathrm{d})$ and TAG $(2.88 \mathrm{~g} / \mathrm{d})$ (highALA-TAG group) or as TAG only $(14.12 \mathrm{~g} / \mathrm{d}$ ) (high-ALATAG group) (Table 1). The $12 \mathrm{~g}$ oil per $\mathrm{d}$ was the identical flaxseed oil in the two groups in order to obtain a standardised and high level of ALA in the diet. The products constituted in total approximately $35 \mathrm{~g}$ fat per d (Table 1), corresponding to about $1255.2 \mathrm{~kJ} / \mathrm{d}(300 \mathrm{kcal} / \mathrm{d})$. The food items were delivered at baseline and after 6 weeks of intervention. At 4 weeks prior to the run-in period and during the intervention 
Table 1. Fatty acid composition in the test products ( $g$ per portion* and g per d)

\begin{tabular}{|c|c|c|c|c|c|c|c|c|}
\hline \multirow[b]{2}{*}{ (g) } & \multicolumn{4}{|c|}{ High ALA-DAG $(n 10)$} & \multicolumn{4}{|c|}{ High ALA-TAG $(n 13)$} \\
\hline & Margarine & Mayonnaise & Oil $\dagger$ & Per d & Margarine & Mayonnaise & Oil $\dagger$ & Per d \\
\hline Total fat & $14 \cdot 08$ & $8 \cdot 84$ & $12 \cdot 0$ & 34.92 & $14 \cdot 12$ & 8.84 & $12 \cdot 00$ & 34.96 \\
\hline DAG & 11.20 & - & - & $11 \cdot 20$ & - & - & - & - \\
\hline TAG & 2.88 & $8 \cdot 84$ & $12 \cdot 0$ & 23.72 & $14 \cdot 12$ & 8.84 & $12 \cdot 00$ & 34.96 \\
\hline SFA & $2 \cdot 80$ & 0.67 & 0.83 & $4 \cdot 30$ & $2 \cdot 80$ & 0.67 & 0.83 & 4.30 \\
\hline MUFA & $7 \cdot 40$ & 5.06 & 1.80 & 14.26 & $7 \cdot 20$ & 5.06 & $1 \cdot 80$ & 14.06 \\
\hline $18: 3 n-3$ (ALA) & 0.78 & 0.79 & 6.72 & 8.29 & 1.00 & 0.79 & 6.72 & 8.51 \\
\hline $20: 5 n-3$ (EPA) & 0.01 & 0.01 & 0.01 & 0.03 & 0.01 & 0.01 & 0.01 & 0.03 \\
\hline $22: 6 n-3$ (DHA) & 0.01 & 0.01 & 0.01 & 0.03 & 0.01 & 0.01 & 0.01 & 0.03 \\
\hline Total $n-3$ & 0.80 & 0.79 & 6.84 & 8.43 & 1.02 & 0.79 & 6.84 & 8.65 \\
\hline $18: 2 n-6$ (LA) & $2 \cdot 20$ & 1.76 & 1.80 & $5 \cdot 76$ & $2 \cdot 20$ & 1.76 & 1.80 & $5 \cdot 76$ \\
\hline Vitamin E & $6 \cdot 20$ & 1.54 & $11 \cdot 32$ & 19.06 & 3.26 & 1.54 & $11 \cdot 32$ & $16 \cdot 12$ \\
\hline
\end{tabular}

ALA, $\alpha$-linolenic acid; DAG, diacylglycerol; LA, linoleic acid.

* One portion of margarine, mayonnaise and oil equals 20,11 and $12 \mathrm{~g}$, respectively.

$\dagger$ Flaxseed oil.

period, the subjects were not allowed to use $n-3$ fatty acid supplements and other dietary supplements. At each visit they were reminded by a nutritionist to keep a stable weight. Fasting body weight and body composition measurements were registered at all visits. The subjects were informed not to change the physical activity and dietary habit during the study. The diet was registered at each visit using the SmartDiet questionnaire which is a validated qualitative dietary questionnaire ${ }^{(29)}$.

Blinding and randomisation. The present study was double-blinded by the identical appearance of different food items; their contents were identifiable only by ID numbers on the food items. Randomisation was performed by LINK Medical Research AS using Microsoft Excel and its random generator.

Compliance. Participants were instructed to bring back food containers (lids of margarine, sachets of mayonnaise and bottles of oil) and compliance was estimated by counting the empty food containers and estimating the leftovers. We estimated compliance by dividing the amount of leftovers by the total amount of food scheduled for the intervention period. The mean compliance for each subject was expressed as a percentage. According to the protocol, subjects with low compliance $(<70 \%)$ were excluded from the study. Compliance was estimated to be $90-100 \%$ in both groups.

\section{Study products}

Margarine and mayonnaise were based on commercial products manufactured by Mills DA. Mayonnaise with $80 \%$ fat was based on refined sunflower oil (Aarhus Karlshamn) for the run-in period and refined rapeseed oil (Aarhus Karlshamn) for the high-ALA groups. Mayonnaise was packed in commercial portion sachets of $11 \mathrm{~g}$.

Margarine with $70 \%$ fat was based on sunflower oil (run-in period), refined rapeseed oil (AarhusKarlshamn AB) (highALA-TAG group) or rapeseed oil with DAG (Kao
Corporation) (high-ALA-DAG group), produced by Danisco AS. The margarine was packed in commercial lids, $140 \mathrm{~g}$ in each.

Refined sunflower oil (AarhusKarlshamn AB) (run-in period) was filled into green, transparent polyethylene ferephthalate bottles of $500 \mathrm{ml}$. Cold pressed flaxseed oil (Norsk Fropresse AS) (high-ALA groups) was fortified with vitamin $\mathrm{E}$ acetate from $\mathrm{BASF}$ in order to obtain equal amounts of vitamin $\mathrm{E}$ for both groups and filled into identical bottles. The participants were told to cover the bottles with aluminum foil and keep the oil refrigerated.

The fatty acid profile and vitamin $\mathrm{E}$ in the products were analysed by the GC method in a routine laboratory (Eurofins). Results are shown in Table 1.

\section{Measurements of body composition}

Subjects wore light clothing and no shoes. Two trained persons carried out all measurements. Height was measured by a wall-mounted stadiometer to the nearest $0.1 \mathrm{~cm}$. Weight was measured by the Tanita BC-418 manufactured by Tanita Corporation to the nearest $0 \cdot 1 \mathrm{~kg}$. To correct for light clothing, $1 \mathrm{~kg}$ was subtracted from the measured value. BMI was calculated by the Tanita BC-418. Waist circumference and hip circumference were measured to the nearest $\mathrm{mm}$ with a standard, non-stretch tape. Waist circumference was measured at the point midway between the iliac crest and the lower rib margin. Hip circumference was measured at the maximum circumference of the buttocks posterior and the symphysis anterior. All subjects were measured while standing in a relaxed position and with normal respiration. Body composition was estimated by bioelectric impedance analysis (Tanita BC-418), a hand-to-foot system, according to the manufacturer's manual. Information on age, height and body type (all were routinely classified as 'standard' body type) was entered before measuring. Bioelectric impedance analysis measurements were carried out at a frequency of $50 \mathrm{kHz}$.

\section{Blood and urine sampling}

The day prior to blood sampling, the subjects were told to refrain from alcohol consumption and vigorous physical 
activity; venous blood samples were drawn after an overnight fast $(\geq 12 \mathrm{~h}$ ). Serum was obtained from silica gel tubes (Becton Dickinson vacutainer) and kept at room temperature for at least $30 \mathrm{~min}$, until centrifugation $(1500 \mathrm{~g}, 12 \mathrm{~min})$. Serum was kept at room temperature and immediately prepared for subsequent analysis of routine laboratory analysis or aliquoted and stored at $-80^{\circ} \mathrm{C}$ until further analyses. Plasma was obtained from EDTA tubes (Becton Dickinson vacutainer), immediately placed on ice and centrifuged within $10 \mathrm{~min}$ $\left(2000 \mathrm{~g}, 4^{\circ} \mathrm{C}\right)$. Plasma samples were aliquoted and stored at $-80^{\circ} \mathrm{C}$ until further analyses. Morning spot urine samples were refrigerated $\left(4^{\circ} \mathrm{C}\right)$ until aliquoted and stored at $-80^{\circ} \mathrm{C}$ until further analysis.

\section{Routine laboratory analysis}

Fasting serum high-sensitivity CRP (hsCRP), total cholesterol, LDL-cholesterol, HDL-cholesterol, TAG, glucose, C-peptide, insulin, HbA1c, thyroid-stimulating hormone, $\boldsymbol{\gamma}$-GT, alkaline phosphatase (ALP), ALAT and aspartame aminotransferase (ASAT) were measured by standard methods at a routine laboratory (Fürst Medical Laboratory). Fasting plasma NEFA were measured by standard methods (Department of Medical Biochemistry, Rikshospitalet University Hospital, Norway).

8 -iso-PGF $2 \alpha$ and creatinine in morning spot urine samples were determined by Vitas AS. Urinary 8-iso-PGF $2 \alpha$ was analysed by liquid chromatography with negative electrospray ionisation coupled to tandem mass spectrometric detection according to Bastani et al. ${ }^{(30)}$. Urinary levels of 8 -iso- $\mathrm{PGF}_{2 \alpha}$ are presented as the ratio of 8 -iso- $\mathrm{PGF}_{2 \alpha}$ to creatinine. Urine creatinine was determined by ELISA using the Cayman Chemical Creatinine Assay kit. The ninety-six-well plate format ELISA kit, validated for urine samples, uses colorimetric detection at $495 \mathrm{~nm}$. Assay range: 0-150 mg/l (normal range 250-4000 $\mathrm{mg} / \mathrm{l})$.

Plasma $\alpha$-tocopherol analysis ${ }^{(31)}$ and quantification of fatty acids in plasma lipids ${ }^{(32)}$ were performed by Vitas AS.

\section{Enzyme immunoassay}

Serum was stored at $-80^{\circ} \mathrm{C}$ in multiple aliquots until analysis. Serum leptin, adiponectin, resistin and fetuin-A were measured by enzyme immunoassay by R\&D Systems according to the manufacturer's instructions.

\section{Statistics}

Sample size was calculated based on the existing literature primarily on the effects of ALA on hsCRP in dietary intervention studies since this outcome measurement would require the highest number of subjects. The calculations used an expected change in hsCRP from baseline to the end of the study of 20 (SD 0.5 ) $\%$. The level of significance was set to $5 \%$ (two-sided) and the power to $80 \%$. A total of seventy-five subjects were required in the main study (twenty-five per arm; three arms). Seventy-five subjects in total were randomised, with forty-nine subjects being randomised to the present two groups. Due to a high withdrawal rate, a total of twenty-three subjects completed the study. Due to sample size, data were analysed with non-parametric tests and are presented as median (2575 percentiles). Differences between the randomisation groups were analysed at baseline and after 12 weeks of intervention (baseline adjusted values). The changes between the two groups or within groups after 12 weeks of intervention were analysed by the Mann-Whitney $U$ test or the Wilcoxon matched-pair signed-rank test, respectively. Bonferroni correction to adjust for multiple testing was performed (adjusted $P \leq$ 0.001). Correlation analysis was performed using Spearman's rank-order correlation. All analyses were performed using SPSS for Windows (version 19.0).

\section{Results}

In the present study, twenty-three healthy, overweight subjects (six women and seventeen men) participated, randomised in two intervention groups. The subjects were 52 (45-62) years, with a median BMI of $30(28-32) \mathrm{kg} / \mathrm{m}^{2}$. At baseline there were no differences in age between the intervention groups.

\section{Body composition}

At baseline there were no significant differences in blood pressure and body composition (total body fat percentage, total body fat mass, trunk fat mass and fat-free mass (FFM) between the intervention groups. Prior to Bonferroni adjustment, there was a significant difference between the two groups in weight, BMI and waist circumference at baseline $(P=0.03,0.05$ and 0.01 , respectively); however, these differences disappeared after Bonferroni adjustment (Table 2).

Since intake of DAG oil has been associated with weight loss, we investigated the effect on weight change. After 12 weeks of intervention, body weight did not change between or within the two groups. However, there was a nonsignificant trend towards an increase in weight in the highALA-DAG group $(P=0 \cdot 07)$. We also investigated the effect on body composition. Prior to Bonferroni adjustment, there was a significant decrease in both total body fat percentage and trunk fat mass within the high-ALA-DAG group from baseline to 12 weeks of intervention $(P=0.04$ and $0 \cdot 05$, respectively) and this decrease was significantly different from the effect observed in the high-ALA-TAG group $(P=$ $0 \cdot 03$ and $0 \cdot 04$, respectively). There was no significant change within or between the groups in total body fat mass. Concomitantly, there was a significant increase in the amount of FFM within the high-ALA-DAG group $(P=0 \cdot 016)$ from baseline to the end of the study and this change in FFM was borderline significant $(P=0.06)$ between the two groups (Table 2). All differences disappeared after Bonferroni adjustment.

\section{Blood pressure}

Systolic and diastolic blood pressure was measured at baseline and after 12 weeks of intervention (Table 2). Prior to 


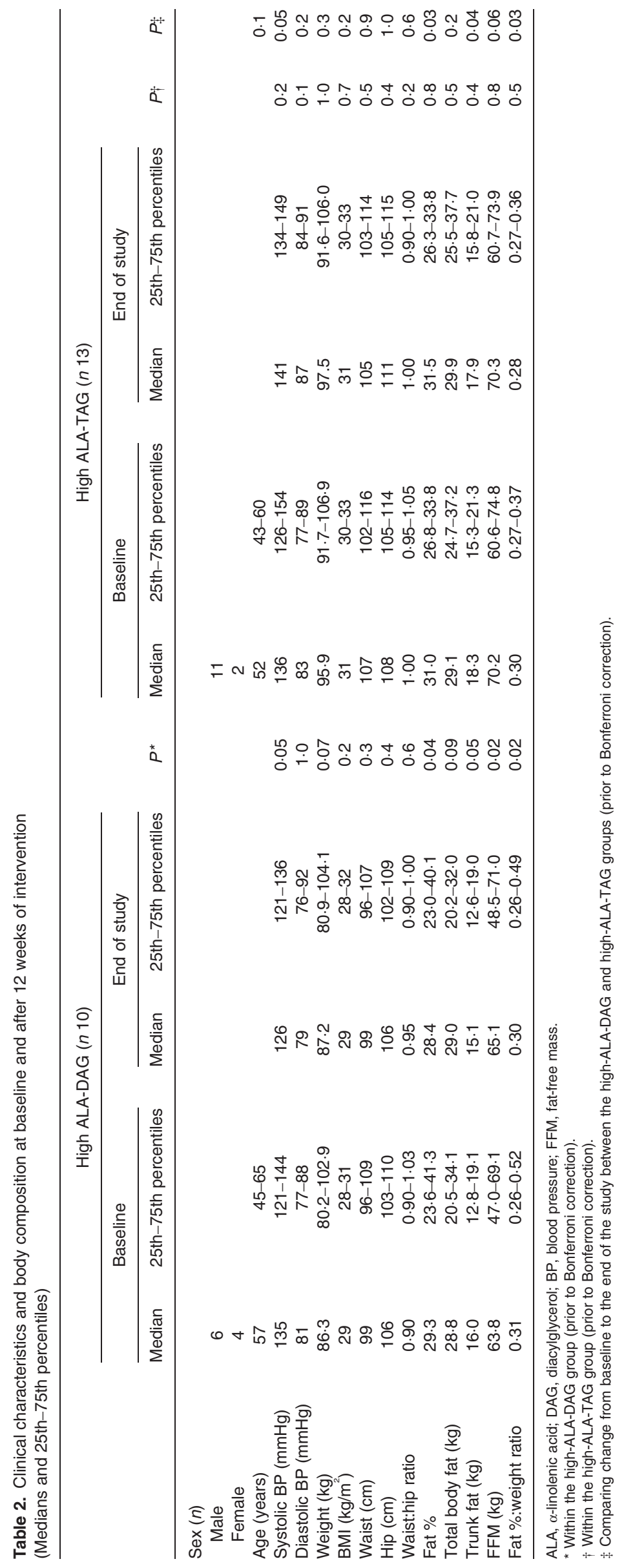


Bonferroni adjustment, there was a significant decrease in systolic blood pressure within the high-ALA-DAG group $(P=$ $0.05)$ after intervention, and the change in systolic blood pressure was significantly different between the two groups $(P=0 \cdot 05)$; however, this was non-significant after Bonferroni adjustment. There was no significant difference in diastolic blood pressure after 12 weeks of intervention between or within any of the groups (Table 2).

\section{Serum lipids, C-reactive protein and insulin sensitivity markers}

At baseline, no differences in serum levels of hsCRP, glucose, insulin, C-peptide, HbA1c, total cholesterol, LDL-cholesterol, HDL-cholesterol and TAG between the intervention groups were observed (Table 3). Baseline values of homeostatic model assessment for insulin resistance (HOMA-IR) were significantly different between the two groups $(P=0 \cdot 05)$. After 12 weeks of intervention, there was no significant difference in the serum hsCRP, glucose, insulin, HOMA-IR, C-peptide, HbA1c, total cholesterol, HDL-cholesterol and TAG between or within the two groups (Table 3). However, after 12 weeks of intervention, there was a non-significant $(P=0.06)$ difference in LDL-cholesterol between the high-ALA groups, with a non-significant decrease in the high-ALA-DAG group (4.0-3.7 mmol/l) and a non-significant increase in the highALA-TAG group (3.3-3.6 mmol/l) (Table 3). All significant differences disappeared after Bonferroni adjustment.

\section{Circulating NEFA}

Circulating NEFA have been shown to be elevated in obese subjects; we therefore measured fasting plasma NEFA concentrations. At baseline, there was no difference in the NEFA concentration between the intervention groups (Table 3). The changes in circulating fasting NEFA concentration were non-significantly (after Bonferroni adjustment) different $(P=0.02)$ between the two groups after 12 weeks of intervention, with a non-significant decrease within the high-ALA-DAG group and a non-significant increase within the high-ALA-TAG group (Table 3).

\section{Liver function parameters}

Obesity leads to accumulation of fatty acids in the liver and therefore we measured markers of liver function such as ASAT, ALAT, $\gamma$-GT and ALP. At baseline no differences in these markers between the intervention groups were observed (Table 4). However, prior to Bonferroni adjustment, the concentration of ALAT, $\gamma$-GT and ALP was significantly reduced within the high-ALA-DAG group $(P=0.02,0.02$ and 0.01 , respectively) and the change in ALAT was significantly different from the high-ALA-TAG group $(P=0.02)$, whereas the change in $\gamma$-GT and ALP was not significantly different from the high-ALA-TAG group (Table 4). These differences disappeared after Bonferroni adjustment. No significant differences within or between the groups in the ASAT:ALAT ratio after 12 weeks of intervention were observed (data not shown). The change in ALAT was positively correlated with the change in trunk fat mass $(r 0.5 ; P=0.007)$ and total body fat mass $(r 0.5 ; P=0.03)$ in the groups calculated together. The change in $\gamma$-GT and ALP did not correlate with the change in total body fat mass or trunk fat mass in any of the groups. In addition, we measured the serum concentration of fetuin- $A$, a suggested marker for hepatic steatosis ${ }^{(33)}$. However, no changes in serum concentration of fetuin-A were found within or between the groups (Table 4).

\section{Adipokines in serum and plasma}

Adipokines are secreted from adipose tissue and since intake of DAG oil has been shown to influence body composition, we measured the circulating hormones leptin, resistin and adiponectin. Despite a non-significant decrease in total body fat percentage and trunk fat mass, no changes between or within any of the groups were observed in any of these hormones after 12 weeks of intervention (Table 5). Also, there were no correlations between the change in hormones and total body fat mass or trunk fat mass.

\section{Oxidative stress markers}

Since ALA has been shown to be rapidly oxidised ${ }^{(34)}$, we measured the oxidative stress markers urinary 8 -iso- $\mathrm{PGF}_{2 \alpha}$ and the antioxidant vitamin $\mathrm{E}$. No significant changes between or within the groups were observed for urinary 8 -iso- $\mathrm{PGF}_{2 \alpha}$ after 12 weeks of intervention (Table 5). Prior to Bonferroni adjustment, the ratio of $\alpha$-tocopherol:total lipids was significantly reduced within the high-ALA-DAG group $(P=0.02)$ and the high-ALA-TAG group $(P=0 \cdot 05)$, with no significant difference between the groups after 12 weeks of intervention (Table 5). These differences disappeared after Bonferroni adjustment.

\section{Fatty acid composition in plasma}

The fatty acid composition in plasma was measured and is shown in Table 6. Prior to Bonferroni adjustment, a significant increase in ALA was observed both within the high-ALADAG $(P=0.005)$ and the high-ALA-TAG groups $(P=$ 0.002 ) (Table 6). The change in plasma ALA between the groups was significantly different $(P=0.04)$ after 12 weeks of intervention, with the largest increase observed in the high-ALA-TAG group. Plasma EPA (20:5n-3) was significantly increased within both groups $(P=0.04$ and 0.02 , respectively), but the change was not significantly different between the groups. No increase was observed in plasma DHA (22: 6n-3) either within or between the two groups. Linoleic acid (18:2n-6) was significantly reduced within both groups ( $P=0.03$ in both groups) after 12 weeks of intervention, but there was no significant difference between them. Arachidonic acid (20:4n-6) was not changed within any of the groups, but a significant change between the two groups $(P=$ $0 \cdot 05)$ was found. Minor changes were observed among the other fatty acids (Table 6). All these differences disappeared after Bonferroni adjustment. 


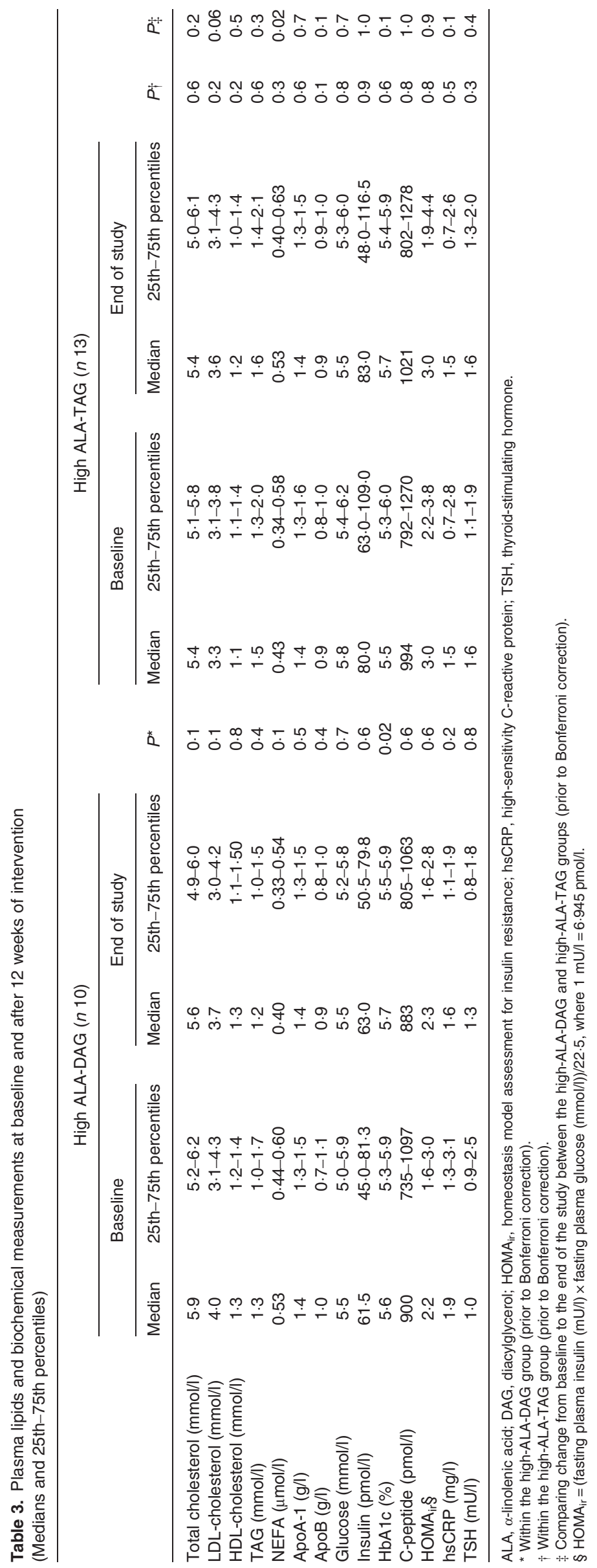


Table 4. Plasma liver function markers at baseline and after 12 weeks of intervention (Medians and 25th-75th percentiles)

\begin{tabular}{|c|c|c|c|c|c|c|c|c|c|c|c|}
\hline & \multicolumn{4}{|c|}{ High ALA-DAG $(n+10)$} & \multicolumn{5}{|c|}{ High ALA-TAG $(n 13)$} & \multirow[b]{3}{*}{$P \dagger$} & \multirow[b]{3}{*}{$P \ddagger$} \\
\hline & \multicolumn{2}{|c|}{ Baseline } & \multicolumn{2}{|c|}{ End of study } & \multirow[b]{2}{*}{$P^{\star}$} & \multicolumn{2}{|c|}{ Baseline } & \multicolumn{2}{|c|}{ End of study } & & \\
\hline & Median & $\begin{array}{l}\text { 25th-75th } \\
\text { percentiles }\end{array}$ & Median & $\begin{array}{l}\text { 25th-75th } \\
\text { percentiles }\end{array}$ & & Median & $\begin{array}{l}\text { 25th-75th } \\
\text { percentiles }\end{array}$ & Median & $\begin{array}{l}\text { 25th-75th } \\
\text { percentiles }\end{array}$ & & \\
\hline ASAT (U/I) & 25.5 & $23 \cdot 0-32 \cdot 3$ & 25.5 & $22 \cdot 8-29 \cdot 3$ & 0.2 & $27 \cdot 0$ & $24.0-28.0$ & $27 \cdot 0$ & $24 \cdot 0-31 \cdot 0$ & 0.3 & 0.1 \\
\hline ALAT (U/I) & 29.0 & $20.5-37.5$ & 24.5 & $19.5-31.5$ & 0.02 & 28.0 & $22 \cdot 5-37 \cdot 0$ & $27 \cdot 0$ & $22 \cdot 0-38 \cdot 0$ & 0.6 & 0.02 \\
\hline$\gamma \mathrm{GT}(\mathrm{U} / \mathrm{I})$ & $24 \cdot 0$ & $18 \cdot 5-32 \cdot 0$ & $19 \cdot 0$ & $14 \cdot 0-28.5$ & 0.02 & $32 \cdot 0$ & $22 \cdot 0-48.5$ & $30 \cdot 0$ & $21.5-63.0$ & 0.6 & 0.3 \\
\hline ALP (U/I) & $71 \cdot 0$ & $63.5-75.0$ & 63.5 & $56 \cdot 3-69 \cdot 3$ & 0.01 & $62 \cdot 0$ & $57 \cdot 0-76 \cdot 0$ & $64 \cdot 0$ & $53 \cdot 0-85.5$ & 0.6 & 0.1 \\
\hline $\begin{array}{l}\text { Fetuin-A } \\
(\mu \mathrm{g} / \mathrm{ml})\end{array}$ & $478 \cdot 2$ & $360 \cdot 0-592 \cdot 6$ & $452 \cdot 2$ & $348 \cdot 1-514 \cdot 2$ & 0.6 & $446 \cdot 6$ & $368 \cdot 8-498 \cdot 6$ & $441 \cdot 8$ & $326 \cdot 2-587 \cdot 6$ & 0.9 & 0.7 \\
\hline
\end{tabular}

ALA, $\alpha$-linolenic acid; DAG, diacylglycerol; ASAT, aspartate aminotransferase; ALAT, alanine aminotransferase; $\gamma$ GT, $\gamma$-glutamyl transpeptidase; ALP, alkaline phosphatase.

* Within the high-ALA-DAG group (prior to Bonferroni correction).

$\dagger$ Within the high-ALA-TAG group (prior to Bonferroni correction).

¥ Comparing change from baseline to the end of the study between the high-ALA-DAG and high-ALA-TAG groups (prior to Bonferroni correction).

\section{Risk of CVD (10-year risk)}

A cardiovascular risk profile has been developed based on the results of the Framingham heart study ${ }^{(35,36)}$ for use in primary care (http://www.reynoldsriskscore.org/). In addition to sex, age, systolic blood pressure, total cholesterol, HDL-cholesterol and hsCRP, the Reynold risk score uses information about smoking and genetic disposure (whether or not a parent had a heart attack before the age of 60 years) to predict CVD risk. In the present study, even though the cardiovascular risk score was relatively low, there was a mean predicted relative risk reduction of $18.5 \%$ (from 6.55 $\%$ to $5.35 \%, P=0.04$ ) in the high-ALA-DAG group, while the high-ALA-TAG group had a relative risk increase of $13.3 \%$ (from $7.53 \%$ to $8.53 \%, P=0.4$ ) after intervention. There was a significant difference in relative risk reduction between the two groups $(P=0 \cdot 001)$. This significant difference remained valid even after full Bonferroni adjustment.

\section{Discussion}

In the present study, we found that replacing part of the TAG-containing margarine with DAG-containing margarine $(20 \mathrm{~g})$ for 12 weeks led to an improved predicted 10 years cardiovascular risk score in healthy, overweight subjects. This reduction in cardiovascular risk may possibly be due to an improved metabolic phenotype appearing as reduced total body fat percentage and reduced trunk fat mass, and increased total FFM, in addition to reduced blood pressure. Additionally, the liver function markers ALAT, $\boldsymbol{\gamma}$-GT and ALP were also non-significantly decreased after DAG intake.

The results are in line with other studies that showed a change in body composition after intake of DAG with a reduction of visceral fat mass ${ }^{(21,37,38)}$ and total fat mass $^{(21,22,38)}$ or total fat percentage ${ }^{(37)}$. The different metabolic effects after intake of DAG compared with those after intake of TAG may be due to their structural differences and not only their fatty acid composition since the energy value, absorptive and digestive properties are similar ${ }^{(39)}$. The main end products of lipase action on $s n-1,3-\mathrm{DAG}$ are free glycerol and $\operatorname{NEFA}^{(40)}$. These end-products of DAG are less readily re-synthesised into chylomicron TAG because such a synthesis requires a glycerol-3-phosphate intermediate. Therefore, fatty acids from DAG are likely to bypass the lymphatic system and enter the blood via the portal vein ${ }^{(41,42)}$ and may hence lead to increased hepatic $\beta$-oxidation, which have been reported both in animal ${ }^{(43-47)}$ and human studies ${ }^{(20,48-51)}$.

Plasma levels of NEFA have been shown to be elevated in obese subjects ${ }^{(52)}$ as a consequence of increased release from the enlarged adipose tissue and reduced clearance ${ }^{(53)}$. NEFA have been shown to inhibit the anti-lipolytic action of insulin, which again will further increase the level of NEFA and subsequently induce insulin resistance ${ }^{(54,55)}$. However, the mechanisms by which obesity and NEFA cause insulin resistance are not clearly understood; nevertheless the reduced release of NEFA into the circulation improves insulin sensitivity ${ }^{(56)}$. In the present study, we found no significant difference in the change in NEFA between the two groups, and no correlation was found between the change in NEFA concentration and the change in insulin resistance markers (insulin, glucose, C-peptide, HbA1c and HOMA-IR) (data not shown).

Despite a non-significant reduction in total body fat percentage and trunk fat mass, total body weight in the high-ALADAG group was not reduced; rather a non-significant increase was observed. Inconsistent results have been described regarding the effect on body weight after intervention with dietary DAG, with some finding a reduced body weight ${ }^{(21,22)}$ while others find no change in weigh ${ }^{(57,58)}$. The lack in weight reduction in the present study may be partly explained by the finding that the amount of FFM was non-significantly increased in the high-ALA-DAG group. The change in FFM in the highALA-DAG group corresponds to a $1.3 \mathrm{~kg}$ or $2 \%$ increase in FFM, thus balancing the potential positive effect of the reduced trunk fat mass. However, taken together, these small nonsignificant changes in body composition may suggest an improvement of the metabolic phenotype in healthy, overweight individuals after dietary DAG intervention.

Even though the total trunk fat mass was slightly reduced in the high-ALA-DAG group, hip circumference was not changed after DAG intervention, indicating the potential effect of DAG intake on visceral fat mass in preference to subcutaneous fat mass. Visceral fat and subcutaneous fat are 
recognised to have distinct metabolic activities ${ }^{(6-9,59)}$, in which adipokines (cytokines and hormones) are secreted in different proportions. While cytokines are mainly secreted from visceral fat, the proportion of secreted hormones is higher from subcutaneous fat ${ }^{(4,60)}$. In the present study, we did not observe any change in the adipocyte hormone concentration (leptin, resistin and adiponectin) which may be partly explained by the lack of reduction in subcutaneous fat depots. In addition, we did not observe any changes in levels of hsCRP in any of the groups after intervention. This is in accordance with Nelson et al., who did not find any change in inflammatory markers, including CRP, after intervention with ALA in overweight, healthy individuals ${ }^{(27,28,61)}$. However, this is in contrast to what others have previously shown regarding the anti-inflammatory effects of ALA ${ }^{(62-64)}$. These studies were also different in duration, population and samples size and thus may be explaining some of the observed discrepancy regarding CRP.

Hypertension is common in obesity ${ }^{(65,66)}$ and a feature of the metabolic syndrome, and is also an established risk factor for $\mathrm{CVD}^{(67)}$. Systolic blood pressure was slightly reduced both within the high-ALA-DAG group and compared with the high-ALA-TAG group. The underlying molecular mechanisms controlling blood pressure are multi-factorial. Weight loss, and in particular reduced visceral fat, is associated with reduced blood pressure ${ }^{(68)}$. The high-ALA-DAG group nonsignificantly reduced the body trunk fat mass after intervention and this small change in body fat may contribute to partly explain the observed improvement in blood pressure in this group, although there was no association between change in body fat and change in systolic blood pressure (data not shown).

In the present study, we did not find any significant changes in serum lipids after DAG intake; only a non-significant reduction in total cholesterol and LDL-cholesterol was found after 12 weeks of intervention with DAG. Others have found effects on plasma lipids after DAG intake; however, these studies have mainly focused on postprandial measurements and/or in groups of patients ${ }^{(18,37,57,69)}$.

Elevated levels of circulating ALAT and $\gamma$-GT, without fatty liver or hepatic dysfunction, in healthy and asymptomatic subjects may predict the future development of metabolic diseases ${ }^{(14,70-73)}$. Furthermore, elevated levels of ALAT and $\gamma$-GT activities have recently been shown to be positively associated with accumulation of visceral fat ${ }^{(12)}$. In the present study, we found that the high-ALA-DAG group non-significantly reduced their circulating concentrations of ALAT, $\gamma-G T$ and ALP after intervention. Also, there was a positive association between the reduction in ALAT concentration and the reduction in body trunk fat mass and total body fat mass. This is in accordance with the findings of Mochizuki et al. and supports the notion that reduction in visceral fat is favourable for improved liver function ${ }^{(12)}$. The present study indicates that fatty acids taken as TAG or DAG will affect liver function in different ways, since there was no association between ALAT and body or trunk fat mass in the TAG group. This differential action on lipid metabolism, explained by specific structural differences of DAG isomers, and not only the fatty acid composition of DAG or TAG, has previously been reported ${ }^{(74)}$. Murase et al. have previously shown that increased hepatic 


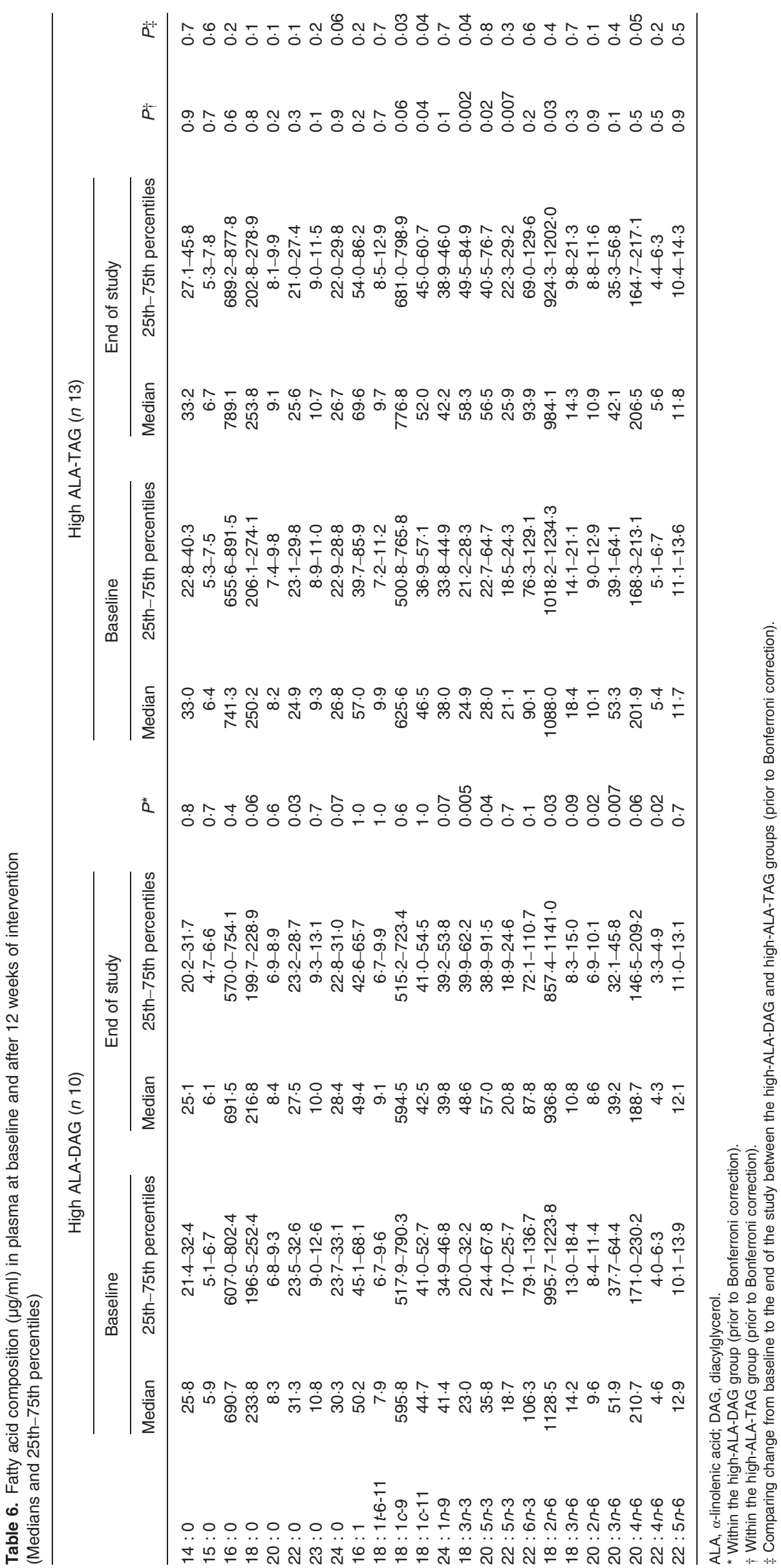


$\beta$-oxidation may be the reason for reduced fatty liver formation after DAG intake ${ }^{(47)}$. Fetuin-A, a marker of hepatic steatosis ${ }^{(33)}$, was not changed within or between the groups after intervention. However, fetuin-A is a marker of a more established disease (hepatic steatosis) while the other liver function markers we measured (ASAT, ALAT, ALP and $\gamma$-GT) reflect a more general hepatic dysfunction. This may partly explain why fetuin-A is not changed during intervention in the present group of healthy, overweight subjects.

The Reynold risk score is a score based on several risk factors of CVD (sex, age, blood pressure, CRP, total cholesterol, HDL-cholesterol, smoking habits and familiar disposal). The risk score gives a CVD prediction for the following 10 years and is intended for use in primary care. In the present study, even though the cardiovascular risk score was relatively low at intervention start, intervention with DAG led to a relative risk reduction of $18.5 \%$ and this reduction was significantly different from the TAG group, supporting the notion of improved health after DAG intake.

The strength of the present study is the randomised controlled design and the high compliance regarding intake of the study products. However, a major weakness in the present study is that the number of participants was considerably reduced due to a high withdrawal rate during the study. In a previous study investigating bioavailability of flaxseed oil, a relatively high withdrawal rate was also observed ${ }^{(75)}$. In the present study, the subjects were randomised and stratified according to sex and age. Due to the high withdrawal rate, however, the final groups had a skewed sex distribution. We therefore recalculated the results based on the male population alone ( $n 6$ and $n 11$ in the high-ALA-TAG and high-ALADAG group, respectively), and the majority of the results still showed the same pattern. Within the high-ALA-DAG group (Wilcoxon matched-pair signed-rank test) a reduction after intervention was found for the following parameters: systolic blood pressure, ASAT, $\gamma$-GT, ALP, Reynold risk score and $\alpha$-tocopherol/total lipids even though not all the recalculated results (only the males) now reached statistical significance. Differences (non-significant) between the two groups (Mann-Whitney $U$ test) were still observed for ALAT, fat percentage and Reynold risk score similarly to the whole group. In the present study, power analysis was based on the existing literature on the effect of ALA on hsCRP, based on the assumption that changes in hsCRP would require the largest sample size. However, in addition to hsCRP, the present study aimed to investigate several other metabolic markers after intake of DAG oil in a Norwegian population, and may therefore be considered exploratory. Due to the limited numbers of subjects and the multiple testing, we performed Bonferroni correction. We cannot rule out the possibility that the results regarding some of the other markers would have been different in a larger population. However, body composition, another outcome in the present study, is shown by others to significantly change with approximately ten subjects in each group ${ }^{(38,76,77)}$, which is comparable to our sample size. In order to further study beneficial health effects of DAG, future studies should include larger sample size and individuals with features of the metabolic syndrome which are more at risk of developing chronic diseases such as fatty liver, CVD and type 2 diabetes mellitus.

In conclusion, the results from the present study show that replacing TAG-based food items with DAG-based food items led to improved predicted 10 years cardiovascular risk score together with a possible beneficial effect on body composition and blood pressure. Even though weight was not reduced, intake of DAG-containing food items seemed to improve the metabolic phenotype in healthy, overweight subjects with small beneficial non-significant changes in total body fat percentage and trunk fat mass, FFM, ALAT, $\gamma$-GT and ALP. Altogether, this may potentially suggest beneficial health effects of DAG in healthy overweight subjects.

\section{Acknowledgements}

The present work was performed at Oslo and Akershus University College of Applied Sciences, Post Box 4, St. Olavsplass, 0130 Oslo, Norway. We thank our research participants for their dedication to the project. We are also grateful to Professor Jan I. Pedersen and Professor Lene Frost Andersen at Department of Nutrition, The Medical Faculty at the University of Oslo for valuable discussions and critical reading of the manuscript, and Ellen Raael at Oslo and Akershus University College of Applied Sciences for valuable assistance with this project. We also thank Danisco AS (Brabrand, Denmark), Norsk fropresse AS (Lysaker, Norway) and Kao Corporation (Tokyo, Japan). This study was supported by Oslo and Akershus University College of Applied Sciences, Norway, The Throne Holst Foundation, The Nordic Center of Excellence on Systems Biology in controlled dietary interventions and cohort studies, SYSDIET (no. 070014), Mills DA, Oslo, Norway, and The Research Council of Norway (P.O. Box 2700, St. Hanshaugen, N-0131 Oslo, Norway). V. H. T.-H., I. N., N. W.-R., K. R., A. M., B. H., K. B. H. and S. M. U. have no conflicts of interest, or any financial or personal interest. L. G. is a researcher and nutrition manager, K. F. C. and A. L. are researchers in Mills DA, Oslo, Norway. They have no financial or personal interest. V. H. T.-H., I. N., K. R., N. W.-R., A. M., L. G., K. F. C., A. L., B. H., K. B. H. and S. M. U. designed the research (project conception, development of overall research plan and study oversight). V. H. T.H., I. N., N. W.-R., K. F. C., A. L., K. B. H. and S. M. U. conducted research (hands-on conduct of the experiments and data collection). V. H. T.-H., K. B. H. and S. M. U. analysed the data or performed statistical analysis. V. H. T.-H., I. N., K. R., N. W.-R., A. M., L. G., K. F. C., A. L., B. H., K. B. H. and S. M. U. wrote the paper (the only authors who made a major contribution). V. H. T.-H., K. B. H. and S. M. U. had primary responsibility for the final content.

\section{References}

1. Rao GH, Thethi I \& Fareed J (2011) Vascular disease: obesity and excess weight as modulators of risk. Expert Rev Cardiovasc Ther $\mathbf{9}$, $525-534$. 
2. Kahn SE, Hull RL \& Utzschneider KM (2006) Mechanisms linking obesity to insulin resistance and type 2 diabetes. Nature 444, 840846.

3. Kotsis V, Stabouli S, Papakatsika S, et al. (2010) Mechanisms of obesity-induced hypertension. Hypertens Res 33, 386-393.

4. Wajchenberg BL (2000) Subcutaneous and visceral adipose tissue: their relation to the metabolic syndrome. Endocr Rev 21, 697-738.

5. Perrini S, Leonardini A, Laviola L, et al. (2008) Biological specificity of visceral adipose tissue and therapeutic intervention. Arch Physiol Biochem 114, 277-286.

6. Kershaw EE \& Flier JS (2004) Adipose tissue as an endocrine organ. I Clin Endocrinol Metab 89, 2548-2556.

7. Galic S, Oakhill JS \& Steinberg GR (2010) Adipose tissue as an endocrine organ. Mol Cell Endocrinol 316, 129-139.

8. Despres JP \& Lemieux I (2006) Abdominal obesity and metabolic syndrome. Nature 444, 881-887.

9. Nedungadi TP \& Clegg DJ (2009) Sexual dimorphism in body fat distribution and risk for cardiovascular diseases. J Cardiovasc Transl Res 2, 321-327.

10. Kishida K, Funahashi T \& Shimomura I (2011) Clinical significance of visceral fat reduction through health education in preventing atherosclerotic cardiovascular disease - lesson from the Amagasaki Visceral Fat Study: a Japanese perspective. Nutr Metab (Lond) 8, 57.

11. Stefan N, Kantartzis K \& Haring HU (2008) Causes and metabolic consequences of fatty liver. Endocr Rev 29, 939-960.

12. Mochizuki K, Miyauchi R, Misaki Y, et al. (2011) Accumulation of visceral fat is positively associated with serum ALT and gamma-GTP activities in healthy and preclinical middle-aged Japanese men. J Nutr Sci Vitaminol (Tokyo) 57, 65-73.

13. Bo S, Gambino R, Durazzo M, et al. (2005) Associations between gamma-glutamyl transferase, metabolic abnormalities and inflammation in healthy subjects from a population based cohort: a possible implication for oxidative stress. World J Gastroenterol 11, 71097117.

14. Vozarova B, Stefan N, Lindsay RS, et al. (2002) High alanine aminotransferase is associated with decreased hepatic insulin sensitivity and predicts the development of type 2 diabetes. Diabetes 51, 18891895.

15. Marchesini G, Brizi M, Bianchi G, et al. (2001) Nonalcoholic fatty liver disease: a feature of the metabolic syndrome. Diabetes $\mathbf{5 0}$, 1844-1850.

16. van Barneveld T, Seidell JC, Traag N, et al. (1989) Fat distribution and gamma-glutamyl transferase in relation to serum lipids and blood pressure in 38-year old Dutch males. Eur I Clin Nutr 43, 809-818.

17. Yanai H, Tomono Y, Ito K, et al. (2007) Diacylglycerol oil for the metabolic syndrome. Nutr J 6, 43.

18. Taguchi H, Watanabe H, Onizawa K, et al. (2000) Double-blind controlled study on the effects of dietary diacylglycerol on postprandial serum and chylomicron triacylglycerol responses in healthy humans. J Am Coll Nutr 19, 789-796.

19. Tada N, Watanabe H, Matsuo N, et al. (2001) Dynamics of postprandial remnant-like lipoprotein particles in serum after loading of diacylglycerols. Clin Chim Acta 311, 109-117.

20. Kamphuis MM, Mela DJ \& Westerterp-Plantenga MS (2003) Diacylglycerols affect substrate oxidation and appetite in humans. Am J Clin Nutr 77, 1133-1139.

21. Nagao T, Watanabe H, Goto N, et al. (2000) Dietary diacylglycerol suppresses accumulation of body fat compared to triacylglycerol in men in a double-blind controlled trial. J Nutr 130, 792-797.

22. Maki KC, Davidson MH, Tsushima R, et al. (2002) Consumption of diacylglycerol oil as part of a reduced-energy diet enhances loss of body weight and fat in comparison with consumption of a triacylglycerol control oil. Am J Clin Nutr 76, 1230-1236.

23. Xie N, Zhang W, Li J, et al. (2011) $\alpha$-Linolenic acid intake attenuates myocardial ischemia/reperfusion injury through antiinflammatory and anti-oxidative stress effects in diabetic but not normal rats. Arch Med Res 42, 171-181.
24. Rodriguez-Leyva D, Dupasquier CM, McCullough R, et al. (2010) The cardiovascular effects of flaxseed and its omega-3 fatty acid, alpha-linolenic acid. Can J Cardiol 26, 489-496.

25. Dewell A, Marvasti FF, Harris WS, et al. (2011) Low- and high-dose plant and marine (n-3) fatty acids do not affect plasma inflammatory markers in adults with metabolic syndrome. J Nutr 141, 2166-2171.

26. de Goede J, Verschuren WM, Boer JM, et al. (2011) Alpha-linolenic acid intake and 10 year incidence of coronary heart disease and stroke in 20,000 middle-aged men and women in the Netherlands. PLoS One 6, e17967.

27. Kaul N, Kreml R, Austria JA, et al. (2008) A comparison of fish oil, flaxseed oil and hempseed oil supplementation on selected parameters of cardiovascular health in healthy volunteers. $\mathrm{J} \mathrm{Am}$ Coll Nutr 27, 51-58.

28. Barcelo-Coblijn G, Murphy EJ, Othman R, et al. (2008) Flaxseed oil and fish-oil capsule consumption alters human red blood cell $n-3$ fatty acid composition: a multiple-dosing trial comparing 2 sources of $n$-3 fatty acid. Am J Clin Nutr 88, 801-809.

29. Svilaas A, Strom EC, Svilaas T, et al. (2002) Reproducibility and validity of a short food questionnaire for the assessment of dietary habits. Nutr Metab Cardiovasc Dis 12, 60-70.

30. Bastani NE, Gundersen TE \& Blomhoff R (2009) Determination of 8-epi PGF(2alpha) concentrations as a biomarker of oxidative stress using triple-stage liquid chromatography/tandem mass spectrometry. Rapid Commun Mass Spectrom 23, 2885-2890.

31. Panfili G, Fratianni A \& Irano M (2003) Normal phase highperformance liquid chromatography method for the determination of tocopherols and tocotrienols in cereals. J Agric Food Chem 51, 3940-3944.

32. Haug IJ, Sagmo LB, Zeiss D, et al. (2011) Bioavailability of EPA and DHA delivered by gelled emulsions and soft gel capsules. Eur J Lipid Sci Technol 113, 137-145.

33. Stefan N, Hennige AM, Staiger H, et al. (2006) Alpha2-Heremans-Schmid glycoprotein/fetuin-A is associated with insulin resistance and fat accumulation in the liver in humans. Diabetes Care 29, 853-857.

34. Arterburn LM, Hall EB \& Oken H (2006) Distribution, interconversion, and dose response of $n-3$ fatty acids in humans. $A m J$ Clin Nutr 83, 1467S-1476S.

35. Ridker PM, Buring JE, Rifai N, et al. (2007) Development and validation of improved algorithms for the assessment of global cardiovascular risk in women: the Reynolds Risk Score. JAMA 297, 611-619.

36. Ridker PM, Paynter NP, Rifai N, et al. (2008) C-reactive protein and parental history improve global cardiovascular risk prediction: the Reynolds Risk Score for men. Circulation 118, 2243-2251.

37. Yanagisawa $Y$, Kawabata T, Tanaka O, et al. (2003) Improvement in blood lipid levels by dietary $s n-1,3$-diacylglycerol in young women with variants of lipid transporters 54T-FABP2 and -493g-MTP. Biochem Biophys Res Commun 302, 743-750.

38. Teramoto T, Watanabe H, Ito K, et al. (2004) Significant effects of diacylglycerol on body fat and lipid metabolism in patients on hemodialysis. Clin Nutr 23, 1122-1126.

39. Taguchi H, Nagao T, Watanabe $H$, et al. (2001) Energy value and digestibility of dietary oil containing mainly 1,3-diacylglycerol are similar to those of triacylglycerol. Lipids 36, 379-382.

40. Hara K, Onizawa K, Honda H, et al. (1993) Dietary diacylglyceroldependent reduction in serum triacylglycerol concentration in rats. Ann Nutr Metab 37, 185-191.

41. Tada N (2004) Physiological actions of diacylglycerol outcome. Curr Opin Clin Nutr Metab Care 7, 145-149.

42. Rudkowska I, Roynette CE, Demonty I, et al. (2005) Diacylglycerol: efficacy and mechanism of action of an anti-obesity agent. Obes Res 13, 1864-1876.

43. Murata M, Ide T \& Hara K (1997) Reciprocal responses to dietary diacylglycerol of hepatic enzymes of fatty acid synthesis and oxidation in the rat. Br J Nutr 77, 107-121.

44. Murase T, Aoki M, Wakisaka T, et al. (2002) Anti-obesity effect of dietary diacylglycerol in C57BL/6J mice: dietary diacylglycerol stimulates intestinal lipid metabolism. J Lipid Res 43, 1312-1319. 
45. Murase T, Nagasawa A, Suzuki J, et al. (2002) Dietary alphalinolenic acid-rich diacylglycerols reduce body weight gain accompanying the stimulation of intestinal beta oxidation and related gene expressions in C57BL/KsJ-db/db mice. J Nutr 132, 3018-3022.

46. Meng X, Zou D, Shi Z, et al. (2004) Dietary diacylglycerol prevents high-fat diet-induced lipid accumulation in rat liver and abdominal adipose tissue. Lipids 39, 37-41.

47. Murase T, Aoki M \& Tokimitsu I (2005) Supplementation with alpha-linolenic acid-rich diacylglycerol suppresses fatty liver formation accompanied by an up-regulation of beta oxidation in Zucker fatty rats. Biochim Biophys Acta 1733, 224-231.

48. Hibi M, Takase H, Yasunaga K, et al. (2008) Greater fat oxidation with diacylglycerol oil consumption for 14 days compared with triacylglycerol oil consumption in overweight men and women. Int J Obes (Lond) 32, 1841-1847.

49. Saito S, Tomonobu K, Hase T, et al. (2006) Effects of diacylglycerol on postprandial energy expenditure and respiratory quotient in healthy subjects. Nutrition 22, 30-35.

50. Hibi M, Takase H, Yasunaga K, et al. (2008) Fat utilization in healthy subjects consuming diacylglycerol oil diet: dietary and whole body fat oxidation. Lipids 43, 517-524.

51. Kimura S, Tsuchiya H, Inage H, et al. (2006) Effects of dietary diacylglycerol on the energy metabolism. Int J Vitam Nutr Res 76, 75-79.

52. Jensen MD, Haymond MW, Rizza RA, et al. (1989) Influence of body fat distribution on free fatty acid metabolism in obesity. J Clin Invest 83, 1168-1173.

53. Bjorntorp P, Bergman H \& Varnauskas E (1969) Plasma free fatty acid turnover rate in obesity. Acta Med Scand 185, 351-356.

54. Boden G (2011) Obesity, insulin resistance and free fatty acids. Curr Opin Endocrinol Diabetes Obes 18, 139-143.

55. Boden G, Chen X, Ruiz J, et al. (1994) Mechanisms of fatty acid-induced inhibition of glucose uptake. J Clin Invest 93, 24382446.

56. Santomauro AT, Boden G, Silva ME, et al. (1999) Overnight lowering of free fatty acids with Acipimox improves insulin resistance and glucose tolerance in obese diabetic and nondiabetic subjects. Diabetes 48, 1836-1841.

57. Yamamoto K, Asakawa H, Tokunaga K, et al. (2001) Long-term ingestion of dietary diacylglycerol lowers serum triacylglycerol in type II diabetic patients with hypertriglyceridemia. J Nutr 131, 3204-3207.

58. Yasunaga K, Glinsmann WH, Seo Y, et al. (2004) Safety aspects regarding the consumption of high-dose dietary diacylglycerol oil in men and women in a double-blind controlled trial in comparison with consumption of a triacylglycerol control oil. Food Chem Toxicol 42, 1419-1429.

59. Vohl MC, Sladek R, Robitaille J, et al. (2004) A survey of genes differentially expressed in subcutaneous and visceral adipose tissue in men. Obes Res 12, 1217-1222.

60. Havel PJ (2004) Update on adipocyte hormones: regulation of energy balance and carbohydrate/lipid metabolism. Diabetes 53, Suppl. 1, S143-S151.

61. Nelson TL, Stevens JR \& Hickey MS (2007) Inflammatory markers are not altered by an eight week dietary alpha-linolenic acid intervention in healthy abdominally obese adult males and females. Cytokine 38, 101-106.

62. Rallidis LS, Paschos G, Liakos GK, et al. (2003) Dietary alpha-linolenic acid decreases $C$ reactive protein, serum amyloid A and interleukin-6 in dyslipidaemic patients. Atherosclerosis 167, 237-242.

63. Zhao G, Etherton TD, Martin KR, et al. (2004) Dietary alphalinolenic acid reduces inflammatory and lipid cardiovascular risk factors in hypercholesterolemic men and women. J Nutr 134, 2991-2997.

64. Bemelmans WJ, Lefrandt JD, Feskens EJ, et al. (2004) Increased alpha-linolenic acid intake lowers C-reactive protein, but has no effect on markers of atherosclerosis. Eur J Clin Nutr 58, 1083-1089.

65. Kotsis V, Stabouli S, Bouldin M, et al. (2005) Impact of obesity on 24-hour ambulatory blood pressure and hypertension. Hypertension 45, 602-607.

66. Stabouli S, Kotsis V, Papamichael C, et al. (2005) Adolescent obesity is associated with high ambulatory blood pressure and increased carotid intimal-medial thickness. J Pediatr 147, 651-656.

67. Stamler J, Stamler R \& Neaton JD (1993) Blood pressure, systolic and diastolic, and cardiovascular risks. US population data. Arch Intern Med 153, 598-615.

68. MacMahon S, Cutler J, Brittain E, et al. (1987) Obesity and hypertension: epidemiological and clinical issues. Eur Heart J 8, Suppl. B, $57-70$.

69. Meguro S, Higashi K, Hase T, et al. (2001) Solubilization of phytosterols in diacylglycerol versus triacylglycerol improves the serum cholesterol-lowering effect. Eur J Clin Nutr 55, 513-517.

70. Kim CH, Park JY, Lee KU, et al. (2009) Association of serum gamma-glutamyltransferase and alanine aminotransferase activities with risk of type 2 diabetes mellitus independent of fatty liver. Diabetes Metab Res Rev 25, 64-69.

71. Ford ES, Schulze MB, Bergmann MM, et al. (2008) Liver enzymes and incident diabetes: findings from the European Prospective Investigation into Cancer and Nutrition (EPIC) Potsdam Study. Diabetes Care 31, 1138-1143.

72. Doi Y, Kubo M, Yonemoto K, et al. (2007) Liver enzymes as a predictor for incident diabetes in a Japanese population: the Hisayama study. Obesity (Silver Spring) 15, 1841-1850.

73. Wannamethee SG, Shaper AG, Lennon L, et al. (2005) Hepatic enzymes, the metabolic syndrome, and the risk of type 2 diabetes in older men. Diabetes Care 28, 2913-2918.

74. Murase T, Mizuno T, Omachi T, et al. (2001) Dietary diacylglycerol suppresses high fat and high sucrose diet-induced body fat accumulation in C57BL/6J mice. J Lipid Res 42, 372-378.

75. Austria JA, Richard MN, Chahine MN, et al. (2008) Bioavailability of alpha-linolenic acid in subjects after ingestion of three different forms of flaxseed. I Am Coll Nutr 27, 214-221.

76. Yamamoto K, Tomonobu K, Asakawa H, et al. (2006) Diet therapy with diacylglycerol oil delays the progression of renal failure in type 2 diabetic patients with nephropathy. Diabetes Care 29, 417-419.

77. Matsuyama T, Shoji K, Watanabe H, et al. (2006) Effects of diacylglycerol oil on adiposity in obese children: initial communication. J Pediatr Endocrinol Metab 19, 795-804. 